\title{
VEGF C-634G polymorphism is associated with protection from isolated ventricular septal defect: case-control and TDT studies
}

Jun $\mathrm{Xie}^{1,2}$, Long $\mathrm{Yi}^{\star, 1}$, Zheng-Feng $\mathrm{Xu}^{\star, 2}, \mathrm{Xu}-\mathrm{Ming} \mathrm{Mo}^{3}$, Ya-Li Hu${ }^{4}$, Dong-Jin Wang ${ }^{5}$, Hao-Zhen Ren ${ }^{1}$, Bing Han $^{1}$, Yong Wang ${ }^{1}$, Chi Yang ${ }^{1}$, Ye-Lin Zhao ${ }^{1}$, Dong-Quan Shi ${ }^{1}$, Yong-Zhong Jiang ${ }^{3}$, Li Shen ${ }^{3}$, Di Qiao ${ }^{1}$, Shi-Lin Chen ${ }^{5}$ and Bao-Jun Yu ${ }^{1}$

${ }^{1}$ Department of Pathology, Nanjing University Medical School, Nanjing, PR China; ${ }^{2}$ Center of Prenatal Diagnosis, Nanjing Maternity and Child Health Hospital, Nanjing Medical School, Nanjing, PR China; ${ }^{3}$ Department of Cardiothoracic Surgery, Nanjing Children's Hospital, Nanjing, PR China; ${ }^{4}$ Department of Obstetrics and Gynecology, Nanjing University Medical School, Nanjing, PR China; ${ }^{5}$ Department of Cardiothoracic Surgery, Nanjing University Medical School, Nanjing, PR China

The ventricular septal defect (VSD) is the most common congenital heart defect and no candidate susceptibility gene has been identified. Endocardial cushion and outflow septal morphogenesis, malalignment of which induces VSD, have been suggested to be mediated by the vascular endothelial growth factor (VEGP). Three single-nucleotide polymorphism (SNP) variants in promoter and $5^{\prime}$-UTR region of the VEGF gene, C-2578A (rs699947), G-1154A (rs1570360) and G-634C (rs2010963), were reported to alter its expression. We assessed the association in a Chinese population between these SNPs and VSD using a double approach: case-control and TDT designs. Among the three SNPs, only $-634 \mathrm{C}$ allele was less frequently present in 222 patients compared to 352 controls (odds ratio: $0.76,95 \%$ Cl: 0.59 $0.97, X^{2}=5.06, P=0.024$, not significant after a Bonferroni correction). This was significantly less transmitted to VSD patients (trios: 142 ) (odds ratio: $0.39,95 \% \mathrm{Cl}: 0.25-0.62, X^{2}=8.11, \mathrm{df}=1, P=0.004$, corrected $P=0.024)$. A similar result was observed for haplotype $-2578 \mathrm{C} /-1154 \mathrm{C} /-634 \mathrm{C}$ allele in both studies (in TDT: $X^{2}=7.51, \mathrm{df}=1, P=0.006$, corrected $P=0.048$ ). All these associations for the first time demonstrated that $-634 \mathrm{C}$ allele was in a significant protective association against VSD, suggesting that VEGF dysregulation was involved in the pathological processes of VSD.

European Journal of Human Genetics (2007) 15, 1246-1251; doi:10.1038/sj.ejhg.5201890; published online 11 July 2007

Keywords: VEGF; VSD; SNP

*Correspondence: Dr L Yi, Department of Pathology, Nanjing University Medical School, Nanjing 210093, PR China.

Tel: + 86-25-83594692; Fax: + 86-25-83686559;

E-mail: yilong@nju.edu.cn

and Z-F Xu, Center of Prenatal Diagnosis, Nanjing Maternity and Child Health Hospital, Nanjing Medical University, Nanjing 210004, PR China. Tel: + 86-25-84461449; Fax: + 86-25-84461449;

E-mail: njxzf@hotmail.com

Received 10 February 2007; revised 7 June 2007; accepted 14 June 2007; published online 11 July 2007

\section{Introduction}

Ventricular septal defect (VSD) is the most common congenital heart defect (CHD) at birth, occurring in not only isolation, but also a frequent component part of more complex cardiac malformations. ${ }^{1}$ Although some genomic and single-gene disorders, such as chromosome 22q11 deletion (Del22q11), 6p deletion (Del6p), NKX2.5, TBX5 and GATA4 mutations, have been found in only a few percentages of cases of VSD, either isolated or as a 
component of complex cardiac malformations, ${ }^{2-5}$ the genetic etiology in the large majority of these cases remains entirely unknown. So far, VSD has been considered to be multigenetic and multi-pathogenic, yet no susceptibility factors have been discovered.

Vascular endothelial growth factor (VEGF) is a multifunctional angiogenic regulator involved in blood vessel formation, mitogenesis, epithelial cell proliferation and endothelial cell survival. ${ }^{6}$ Dysregulated VEGF expression has been implicated in the pathogenesis of a number of diseases. $^{7-9}$ VEGF plays an important role in embryonic heart development as well, although the detailed function of VEGF remains unclear at present. Previous studies in animal models have revealed two possible mechanisms of the dysregulation of VEGF leading to VSD. Some studies suggested that altered $V E G F$ expression, leading to either over or under production of $V E G F$, may interrupt endocardial cushion development, and contribute to chamber septation and valvular defects. ${ }^{10,11}$ The others showed $V E G F$, as a modifier, interacting with the Tbx1 gene to mediate pharyngeal apparatus patterning, including outflow septum development, a disorder of which resulted in Del22q11 syndrome-like cardiac defects. ${ }^{12}$

Genetic association studies have identified several singlenucleotide polymorphisms (SNPs) that are associated with variations in VEGF protein production. Three of them, C-2578A, G-1154A and G-634C (or G + 405C) have been reported to confer an increased risk for Tetralogy of Fallot $(\mathrm{TOF})^{13}$ and valvuloseptal heart defects. ${ }^{14}$ Two SNPs (positions 2578 and 1154) are located in VEGF promoter which are relative to the $V E G F$ transcription start site, ${ }^{15}$ and one SNP (position -634) in $5^{\prime}$-UTR that affects VEGF expression at both transcription and translation levels. ${ }^{16}$ Taken together, these results indicated VEGF was involved in the development of endocardial cushion and outflow septum. Malalignment of the three septal components (the outflow septum, the ventricular septum and atrioventricular endocardial cushion tissue) could cause a VSD in the perimembranous region. ${ }^{17}$ Our previous studies, as well as other's, have noticed that perimembranous VSD may occur as one of the clinical manifestations of the Del22q11 syndrome. ${ }^{5,18}$ Therefore, we speculated VEGF dysregulation might contribute to the pathological processes of VSD.

This study is aimed at determining whether SNPs within the regulatory region of the VEGF gene were associated with isolated VSD. To achieve this, genotyping and haplotype analysis for VEGF C2578A, G1154A, G-634C SNPs were performed by case-control and transmission disequilibrium test (TDT) studies.

\section{Materials and methods Subject}

A total of 222 affected children with isolated perimembranous VSD, confirmed by cardiac surgery, and 352 unrelated healthy persons were included in the casecontrol study. Both parents were available for 142 of the 222 affected children, so that we could perform a familybase association study with them by means of a TDT. All the affected children (55 girls and 167 boys, aged 2 months to 15 years) had no Del22q11 or other chromosomal abnormalities by our previous analysis. ${ }^{18,19}$ Each family trio was checked for Mendelian transmission by genotyping high informative microsatellites. After informed consent was obtained, blood was drawn or buccal cell was removed with a swab from the subjects for DNA analysis under the supervision of the Medical School of Nanjing University, Nanjing Maternity and Child Health Hospital and Nanjing Children's Hospital.

\section{Genotyping}

Genomic DNA was prepared from peripheral blood leukocytes by using the Chelex-100 method $^{20}$ or from buccal swabs by using the DNA IQ system (Promega, USA), according to the manufacturer's instructions.

Three SNPs in the VEGF gene regulatory regions were carried out by PCR-RFLP analysis. The PCR primers for C-2578A (rs699947), G-1154A (rs1570360) and G-634C (rs2010963) were 5'-GAGGATGGGGCTGACTAGGT-3' (forward) and 5'-TGGTTTCTGACCTGGCTATTTC- $3^{\prime}$ (reverse); $5^{\prime}$-CTGCTCCCTCCTCGCCAATG-3' (forward) and $5^{\prime}$ CGGGGACAGGCGAGCGTCAG-3' (reverse with a mismatch nucleotide, which was italicized to abolish a restriction enzyme site); 5'-TTGCCATTCCCCACTT GAATCG-3' (forward) and 5'-CCGTCAGCGCGACTGGT CA-3' (reverse), respectively.

The reaction mixture was subjected to denaturation at $95^{\circ} \mathrm{C}$ for $2 \mathrm{~min}$, followed by 30 cycles at $94^{\circ} \mathrm{C}$ for $1 \mathrm{~min}$, $60-62^{\circ} \mathrm{C}$ for $1 \mathrm{~min}, 72^{\circ} \mathrm{C}$ for $1 \mathrm{~min}$, then by a final extension at $72^{\circ} \mathrm{C}$ for $10 \mathrm{~min}$. The $-2578 \mathrm{~A},-1154 \mathrm{G}$ and $-634 \mathrm{G}$ alleles resulted in the gain of a BglII, MnlI and BsmFI site, respectively. After digestion by an appropriate restriction enzyme, PCR products were electrophoresed on a $2 \%$ agarose gel and visualized by ethidium bromide staining.

\section{Measurement of plasma $V E G F$ concentrations}

Venous blood samples were taken from 88 healthy subjects before breakfast and drawn into vacuum tubes containing EDTA. Blood samples were quickly centrifuged and plasma fractions were immediately stored at $-80^{\circ} \mathrm{C}$ until analysis. Plasma concentrations of $V E G F$ were measured using a Human VEGF Quantikine Immunoassay (R\&D Systems, USA). In this study, plasma $V E G F$ levels were measured instead of serum levels. VEGF levels in serum are highly variable because blood platelets release a lot of $V E G F$ into the serum after collection. ${ }^{21}$

\section{Statistical analysis}

Hardy-Weinberg equilibrium was tested by a goodnessof-fit $\mathrm{X}^{2}$ test with one degree of freedom. $\mathrm{X}^{2}$ analysis was 
used to evaluate case-control differences in the distribution of allele and genotype. Haplotype analysis in the cases and controls as well as the D' value of Linkage disequilibrium (LD) between paired SNPs were estimated using online software SHEsis (http://202.120.7.14/analysis/myAnalysis.php.). ${ }^{22}$ And TDT analysis was estimated by software GeneHunter. ${ }^{23}$ Given the multiple-comparison problem presented by the data, Bonferroni correction was applied. The coefficient of Bonferroni correction in allele and genotype analysis is 6 (for comparisons of three markers in both TDT and the case-control tests), and in haplotype analysis is 8 (for four haplotypes comparisons in both TDT and case-control tests).

The Student's $t$-test was used to compare the mean levels of plasma $V E G F$ among the G-634C genotypes using an online software (http://www.physics.csbsju.edu/stats/ t-test.html). Significance was considered at $P<0.05$.

\section{Results}

The genotype frequencies of these three polymorphisms of the $V E G F$ gene did not show a significant deviation from the Hardy-Weinberg expectation. The distributions of alleles and genotypes are summarized in Tables 1 and 2 . Although a difference in the distribution of the $\mathrm{G}-634 \mathrm{C}$ variations between the affected children and the control group, this was not statistically significant after Bonferroni correction. The results of alleles and genotypes in TDT are shown in Table 3. TDT analysis of individual SNPs revealed significantly decreased transmission of allele C at G-634C SNP even after Bonferroni correction $\left(\mathrm{X}^{2}=8.11, \mathrm{df}=1\right.$, $P=0.004$, corrected $P=0.024$, odds ratio: $0.39,95 \% \mathrm{CI}$ : $0.25-0.62)$.

Calculated LD coefficient, which showed that there was a strong LD between the polymorphisms -2578 and -1154 $\left(\mathrm{D}^{\prime}=0.99, r^{2}=0.60\right)$; between the polymorphisms -1154 and $-634\left(\mathrm{D}^{\prime}=1, r^{2}=0.14\right)$; and between the polymorphisms -2578 and $-634\left(\mathrm{D}^{\prime}=0.99, \mathrm{r}^{2}=0.22\right)$. The study then inferred Haplotypes of three VEGF polymorphisms and compared their frequencies. Only four of the eight possible haplotypes occurred at an appreciable frequency (a frequency of more than 0.03) in both affected children and controls, which is also shown in Table 4. No significant difference of haplotype was found after Bonferroni correction, although $-2578 \mathrm{C},-1154 \mathrm{G}$ and $-634 \mathrm{C}$ allele (haplotype CGC) was present less frequently in isolated VSD patients. The analysis of 3-SNP haplotype in TDT is shown in Table 5. Haplotype CGC was frequently transmitted to affected children with isolated VSD less frequently than expected by mendelian inheritance $\left(X^{2}=7.51, \mathrm{df}=1\right.$, $P=0.006)$, and haplotype CGG was more transmitted $\left(\mathrm{X}^{2}=7.91, \mathrm{df}=1, P=0.005\right)$. Both two haplotypes remained significant after Bonferroni correction (corrected $P$-values were 0.048 and 0.040 for haplotypes CGC and CGG, respectively).

The study then looked at VEGF plasma levels and VEGF genotypes in 88 healthy subjects to assess the possible functional relevance of the VEGF polymorphisms. There were no significant differences in mean VEGF plasma levels between healthy subjects with the CC genotype of the G-634C polymorphism and those with the GG plus GC genotype $(t=1.08, P=0.28)$. The Figure 1 about comparison of VEGF serum levels with VEGF genotypes was in the

Table 2 Results of genotype analysis in case and control groups

\begin{tabular}{lccccc}
\hline SNPS & VSD $(\mathrm{n}=222)$ & Control $(\mathrm{n}=352)$ & $X^{2}$ & Fisher's $\mathrm{P}$ & $\mathrm{P}_{c}$ \\
\hline C-2578A & & & 1.47 & 0.48. & $\mathrm{NS}$ \\
CC & $124(0.56)$ & $211(0.60)$ & & & \\
CA & $83(0.37)$ & $124(0.35)$ & & & \\
AA & $15(0.068)$ & $17(0.048)$ & & & \\
G-1154A & & & 0.35 & 0.84 & NS \\
GG & $156(0.70)$ & $255(0.72)$ & & & \\
GA & $60(0.27)$ & $89(0.25)$ & & & \\
AA & $6(0.027)$ & $8(0.023)$ & & & \\
G-634C & $78(0.35)$ & $103(0.29)$ & & & \\
GG & $118(0.53)$ & $181(0.51)$ & & & \\
GC & $26(0.12)$ & $68(0.19)$ & & & \\
CC & &
\end{tabular}

NS, not significant; $P_{\mathrm{c}}, P$-value after applying Bonferroni correction.

Table 1 Results of allele analysis in case and control groups

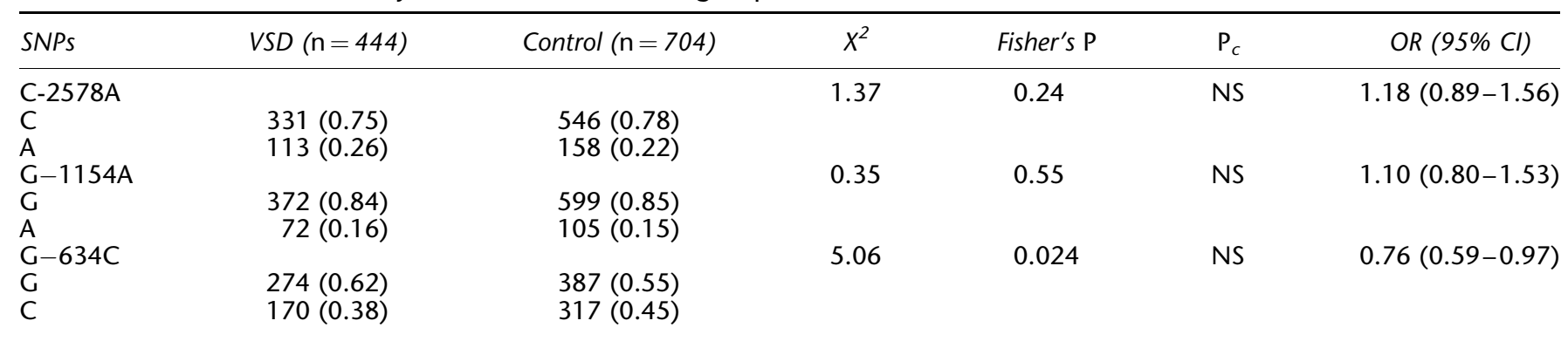

NS, not significant; $P_{\mathrm{c}}, P$-value after applying Bonferroni correction. 
Table 3 Results of TDT analysis in the VSD families $(n=142)$

\begin{tabular}{lccccccc}
\hline SNP & Allele & Transmitted & Untransmitted & $X^{2}$ & P-value & $P_{c}$ & OR (95\% CI) \\
\hline C-2578A & C & $53(0.48)$ & $57(0.52)$ & 0.15 & 0.7 & NS & $0.86(0.51-1.47)$ \\
G-1154A & A & $57(0.52)$ & $53(0.48)$ & 0.15 & 0.7 & NS & NS (Ref.) \\
& G & $38(0.52)$ & $35(0.48)$ & 0.12 & 0.73 & $1.17(0.61-2.26)$ \\
G-634C & G & $35(0.48)$ & $38(0.52)$ & 0.12 & 0.73 & NS (Ref.) & 1 (Ref.) \\
& G & $93(0.62)$ & $58(0.38)$ & 8.11 & 0.004 & 0.024 & $0.39(0.25-0.62)$ \\
\hline
\end{tabular}

NS, not significant; $P_{\mathrm{c}}, P$-value after applying Bonferroni correction.

Table 4 Results of hapolotype analysis in case and control groups

\begin{tabular}{|c|c|c|c|c|c|c|}
\hline$-2578 /-1154 /-634$ haplotype & VSD $(\mathrm{n}=444)$ & Control $(\mathrm{n}=704)$ & $x^{2}$ & Fisher's P & $P_{c}$ & OR $(95 \% \mathrm{Cl})$ \\
\hline CGG & $161.00(0.36)$ & $228.36(0.33)$ & 1.78 & 0.18 & NS & $1.19(0.92-1.52)$ \\
\hline CGC & $170.00(0.38)$ & $315.46(0.45)$ & 4.75 & 0.029 & NS & $0.76(0.60-0.97)$ \\
\hline AGG & $41.00(0.092)$ & $53.64(0.076)$ & 0.94 & 0.33 & NS & $1.23(0.81-1.89)$ \\
\hline AAG & $72.00(0.16)$ & $102.82(0.15)$ & 0.55 & 0.46 & NS & $1.13(0.82-1.57)$ \\
\hline Others & $0.01(0.000)$ & $3.72(0.005)$ & & & & \\
\hline
\end{tabular}

NS, not significant; $P_{\mathrm{c}}, P$-value after applying Bonferroni correction.

Table 5 Transmission of VEGF haplotype to affected offspring $(n=142)$

\begin{tabular}{|c|c|c|c|c|c|c|}
\hline SNP & Haplotype & Transmitted & Untransmitted & $x^{2}$ & P-value & $P_{c}$ \\
\hline$-2578 /-1154 /-634$ & $\begin{array}{l}\text { CGG } \\
\text { CGC } \\
\text { AGG } \\
\text { AAG } \\
\text { Others }\end{array}$ & $\begin{array}{c}52(0.66) \\
32(0.32) \\
21(0.54) \\
24(0.45) \\
0\end{array}$ & $\begin{array}{c}27(0.34) \\
58(0.64) \\
18(0.46) \\
20(0.45) \\
6\end{array}$ & $\begin{array}{l}7.91 \\
7.51 \\
0.23 \\
0.36\end{array}$ & $\begin{array}{l}0.005 \\
0.006 \\
0.63 \\
0.55\end{array}$ & $\begin{array}{l}0.04 \\
0.048 \\
\text { NS } \\
\text { NS }\end{array}$ \\
\hline
\end{tabular}

NS, not significant; $P_{\mathrm{c}}, P$-value after applying Bonferroni correction.

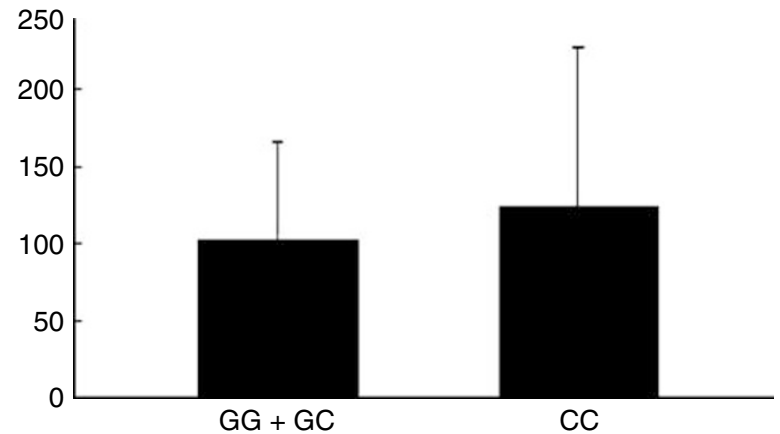

Figure 1 Comparison of VEGF serum levels of 88 healthy subjects with VEGF genotypes. Bars indicate mean values \pm SE. $P=0.28$ (CC vs CG plus GG).

online supplement. Analysis of these 88 subjects revealed that there was no relationship between genetic variants and VEGF plasma levels.

\section{Discussion}

VSD is considered as multifactorial in origin, with various genes interacting with each other or with environmental factors, which makes it hard to detect the susceptibility genes. Therefore, a double approach was used: casecontrol and TDT tests, to make the result more reliable. Case-control test were based on the assumption that any noted differences in allele frequencies actually relate to the outcome measured. Unfortunately, a small portion of differences in allele frequencies between cases and controls are attributable to diversity in background population, which is called population stratification, unrelated to outcome status. TDT as a new linkage method that requires an affected individual and his or her parents, and uses the mendelian principle that, for any polymorphic marker, each parent contributing one allele to an offspring. TDT simply involves the establishment of a pseudo casecontrol study, in which cases are the parental alleles transmitted to the affected proband, and controls are those that were not transmitted. Protection from stratification comes from the matching of each case-control pair within a family, so that any population-level allele frequency differences become irrelevant. As a result, TDT have been used here to avoid spurious association contributed to population stratification. ${ }^{24}$

The study demonstrated that in TDT, $-634 \mathrm{C}$ allele was associated with protection from isolated VSD. The result 
indicated $-634 \mathrm{C}$ allele is significantly less transmitted in affected children, and both OR and 95\% CI values are less than one. In haplotype analysis, significant transmission disequilibrium was observed for haplotypes CGC and CGG. Both of the haplotypes differed only in the G-634C polymorphism. As a result, G-634C SNP was identified responsible for the significant haplotype variations in the finding. These results indicated $-634 \mathrm{C}$ allele is a preventive factor, neutralizing predisposing alleles effects and moving carriers away from the disease-liability threshold.

In the case-control test, marginal association of G-634C SNP and G-634C SNP related haplotype was detected, but not was statistically significant after a Bonferroni correction. The marginal association in casecontrol test may be caused by the small sample size. Due to the number of tests in this study, correction for multiple testing was necessary to reduce type 1 error. However, this reduces the power to detect association and may result in

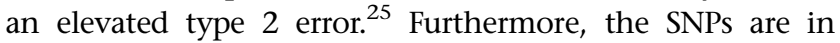
moderate LD, the degree of independence between markers is low. Hence, applying Bonferroni correction is overly conservative, but it should be noted transmission disequilibrium of $\mathrm{G}-634 \mathrm{C}$ variations remained significant despite this.

No relevance was found between $\mathrm{G}-634 \mathrm{C}$ variation and plasma $V E G F$ level among the healthy control group. In previous studies, the relationship between $\mathrm{G}-634 \mathrm{C}$ polymorphism and plasma $V E G F$ level revealed controversial results. Berrahmoune et $\mathrm{al}^{2} \mathrm{~s}^{26}$ heritability study of the health France Stanislas family showed that plasma VEGF concentrations were under genetic control in healthy families, but $\mathrm{G}-634 \mathrm{C}$ polymorphism was not significant in association. Watson et $a l^{27}$ found that higher VEGF production from lipopolysaccharide (LPS) stimulated peripheral blood mononuclear cell in GG homozygotes than in CC homozygotes, and LPS may work through the myeloid zinc-finger protein (MZF1)-binding site, within which the -634 (same as +405 ) polymorphism was located. However, the highest VEGF serum levels were measured in subjects with CC homozygotes by Awata et al. ${ }^{28}$ Because plasma VEGF levels were influenced by many factors such as genetic predispositions, tissue-specific and timespecific, no variation of plasma VEGF with $-634 \mathrm{G}$ allele in the population did not mean that local plasma VEGF levels with -634 polymorphism in cardiac tissue in the period of embryonic heart morphogenesis did not change. Therefore, the apparent inconsistency among studies may be partly caused by methodological differences, especially the difference between in vivo and in vitro studies, as well as spatiotemporal effects of $V E G F$ polymorphisms.

In addition, more and more evidences have shown VEGF expression is affected by the $-634 \mathrm{C}$ to $\mathrm{G}$ substitution. Awata et $a l^{16}$ discovered transcriptional activity in clone containing the $-1154 \mathrm{G} /-634 \mathrm{C}$ haplotype was greater than clones containing the other haplotypes in both a human glioma cell line and human lymphoblastic T-lymphocyte cell line. Another study showed -634G was a risk allele affecting $V E G F$ gene expression. Becoming an element that regulates $V E G F$, internal ribosomal entry site B (IRES-B) was affected by the $-634 \mathrm{G}$ allele. In hypoxic condition, the IRES-B-driven gene expression was found to be increased by $-634 \mathrm{C}$ allele, but not by $-634 \mathrm{G}$ allele. In vitro study, the relative IRES-B activity with the $-634 \mathrm{G}$ allele was $16 \%$ lower in normoxia and $23 \%$ lower in hypoxia than with $-634 \mathrm{C}$ allele. $^{29}$

During the embryonic period, a low level of VEGF expression would result in severe abnormalities in the developing heart. The loss of one VEGF allele which causes low VEGF transcription level induced endocardial cushions and chamber malformations in addition to vascular development disorder. ${ }^{30}$ In Stalmans et al's ${ }^{12}$ study, DGSlike phenotype including VSD and low-level TBX-1 mRNA were observed In VEGF164-deficient mice. In a TBX1 knockdown zebrafish, VEGF knockdown increased the penetrance of pharyngeal arch artery defects in a dosedependent manner. In summary, normal VEGF expression tightly controlled with a narrow window was important to heart morphogenesis.

The study discovered G-634C SNP in VEGF was associated with isolated VSD. Additionally, two genetic association studies, also demonstrated positive relevance between $\mathrm{G}-634 \mathrm{C}$ polymorphism and CHD. Interestingly, $-634 \mathrm{C}$ allele reduced the risk for isolated, nonsyndrome TOF in Lambrechts et al's ${ }^{13}$ study and isolated VSD in the present study. This allele was increased in congenital valvuloseptal heart defects in Vannay et al's ${ }^{14}$ study. Unfortunately, no detailed clinical information revealed, especially whether isolated VSD was included. It is possible that the inconsistency may be explained by different CHD types enrolled in different studies. Furthermore, there may be more than one mechanism of dysregulation of VEGF leading to cardiac malformation.

In summary, the study has identified that the variant $\mathrm{C}$ allele of the - G634C SNP protects against isolated VSD via the stabilizing expression of VEGF. This genetic association suggested VEGF dysregulation was involved in the pathological processes of VSD.

\section{Acknowledgements}

We thank Professor Takahiro Nakamura from National Defense Medical College in Japan for statistic help and Miss Marilyn White from Brigham Young University in USA for Language editing of the manuscript. This work was supported by grants from the Nanjing Science and Technology Development Program (ZKX0312), the Preresearch Foundation of Nanjing University and the Jiangsu Top Expert Program in Six Professions.

\footnotetext{
References

1 Hoffman JI, Kaplan S: The incidence of congenital heart disease. J Am Coll Cardiol 2002; 39: 1890-1900.
} 
2 Garg V, Kathiriya IS, Barnes R et al: GATA4 mutations cause human congenital heart defects and reveal an interaction with TBX5. Nature 2003; 424: 443-447.

3 Benson DW, Silberbach GM, Kavanaugh-McHugh A et al: Mutations in the cardiac transcription factor NKX2.5 affect diverse cardiac developmental pathways. J Clin Invest 1999; 104: $1567-1573$.

4 Mirza G, Williams RR, Mohammed S et al: Refined genotypephenotype correlations in cases of chromosome $6 \mathrm{p}$ deletion syndromes. Eur J Hum Genet 2004; 12: 718-728.

5 Yamagishi H, Maeda J, Tokumura M et al: Ventricular septal defect associated with microdeletions of chromosome 22q11.2. Clin Genet 2000; 58: 493-496.

6 Ferrara N, Gerber HP, LeCouter J: The biology of VEGF and its receptors. Nat Med 2003; 9: 669-676.

7 Fukumura D, Xavier R, Sugiura T et al: Tumor induction of VEGF promoter activity in stromal cells. Cell 1998; 94: 715-725.

8 Aiello LP, Avery RL, Arrigg PG et al: Vascular endothelial growth factor in ocular fluid of patients with diabetic retinopathy and other retinal disorders. N Engl J Med 1994; 331: 1480-1487.

9 Kim SH, Choi YM, Choung SH et al: Vascular endothelial growth factor gene $+405 \mathrm{C} / \mathrm{G}$ polymorphism is associated with susceptibility to advanced stage endometriosis. Hum Reprod 2005; 20: 2904-2908.

10 Dor Y, Camenisch TD, Itin A et al: A novel role for VEGF in endocardial cushion formation and its potential contribution to congenital heart defects. Development 2001; 128: 1531-1538.

11 Miquerol L, Langille BL, Nagy A: Embryonic development is disrupted by modest increases in vascular endothelial growth factor gene expression. Development 2000; 127: 3941-3946.

12 Stalmans I, Lambrechts D, De Smet F et al: VEGF: a modifier of the del22q11 (DiGeorge) syndrome? Nat Med 2003; 9: 173-182.

13 Lambrechts D, Devriendt K, Driscoll DA et al: Low expression VEGF haplotype increases the risk for tetralogy of Fallot: a family based association study. J Med Genet 2005; 42: 519-522.

14 Vannay A, Vasarhelyi B, Kornyei M et al: Single-nucleotide polymorphisms of VEGF gene are associated with risk of congenital valvuloseptal heart defects. Am Heart J 2006; 151: $878-881$.

15 Brogan IJ, Khan N, Isaac K et al: Novel polymorphisms in the promoter and $5^{\prime}$ UTR regions of the human vascular endothelial growth factor gene. Hum Immunol 1999; 60: 1245-1249.

16 Awata T, Kurihara S, Takata N et al: Functional VEGF G-634C polymorphism is associated with development of diabetic macular edema and correlated with macular retinal thickness in type 2 diabetes. Biochem Biophys Res Commun 2005; 333: 679-685.

17 Kirby ML, Waldo KL: Neural crest and cardiovascular patterning. Circ Res 1995; 77: 211-215.

$18 \mathrm{Yi} \mathrm{L}, \mathrm{Xu} \mathrm{ZF}, \mathrm{Mo} \mathrm{XM}$ et al: New tetranucleotide STRP markers for detecting the 22q11.2 deletion. Mol Cell Probes 2006; 20: $359-365$.

19 Zhang XQ, Xu ZF, Yi L et al: Rapid detection of the 22ql 1 microdeletion with quantitative real-time polymerase chain reaction. J Clin Pediatr 2006; 21: 1409-1410, 1422.

20 Walsh PS, Metzger DA, Higuchi R: Chelex 100 as a medium for simple extraction of DNA for PCR-based typing from forensic material. Biotechniques 1991; 10: 506-513.

21 Webb NJ, Bottomley MJ, Watson CJ et al: Vascular endothelia growth factor (VEGF) is released from platelets during blood clotting: implications for measurement of circulating VEGF levels in clinical disease. Clin Sci 1998; 94: 395-404.

22 Shi YY, He L: SHEsis, a powerful software platform for analyses of linkage disequilibrium, haplotype construction, and genetic association at polymorphism loci. Cell Res 2005; 15: 97-98.

23 Kruglyak L, Daly MJ, Reeve-Daly MP et al: Parametric and nonparametric linkage analysis: a unified multipoint approach. Am J Hum Genet 1996; 58: 1347-1363.

24 Cardon LR, Palmer LJ: Population stratification and spurious allelic association. Lancet 2003; 361: 598-604.

25 Perneger TV: What's wrong with Bonferroni adjustments. BMJ 1998; 316: 1236-1238.

26 Berrahmoune $\mathrm{H}$, Herbeth B, Lamont JV et al: Heritability for plasma VEGF concentration in the Stanislas family study. Ann Hum Genet 2007; 71: 54-63.

27 Watson CJ, Webb NJ, Bottomley MJ et al: Identification of polymorphisms within the vascular endothelial growth factor (VEGF) gene: correlation with variation in VEGF protein production. Cytokine 2000; 12: 1232-1235.

28 Awata $\mathrm{T}$, Inoue $\mathrm{K}$, Kurihara $\mathrm{S}$ et al: A common polymorphism in the $5^{\prime}$-untranslated region of the VEGF gene is associated with diabetic retinopathy in type 2 diabetes. Diabetes 2002; 51 : $1635-1639$.

29 Lambrechts D, Storkebaum E, Morimoto $\mathrm{M}$ et al: VEGF is a modifier of amyotrophic lateral sclerosis in mice and humans and protects motoneurons against ischemic death. Nat Genet 2003; 34: 383-394

30 Carmeliet P, Ferreira V, Breier G et al: Abnormal blood vessel development and lethality in embryos lacking a single VEGF allele. Nature 1996; 380: 435 - 439 . 